\title{
A MINIMUM-PHASE FIR COMPLEX FILTER DESIGN FOR TRANSMULTIFLEXER IMPLEMENTATION
}

\author{
E. Del Re $e^{\circ}$ P.L. Emiliani ${ }^{\circ}$, D.F. Maffucci ${ }^{\circ}$ \\ - Istituto di Elettronica, Università di Firenze, \\ Via S. Marta 3, 50139 Firenze, Italy. \\ - Istituto di Ricerca sulle onde Elettromagnetiche, C.N.R., \\ Via Panciatichi 64, 50127 Firenze, Italy.
}

\section{ABSTRACT}

A digital per-channel transmultiplexer system, based on the generation of a baseband analytic signal and on its successive interpolation and filtering, is considered.

The method employs complex filters. To meet the CCITT specifications on the conversion system group delay, minimum-phase FIR complex filters are required. Their design is described in this paper.

The system design also includes the evaluation of the wordlengths of the filter coefficients and the arithmetic operations. Computer simulations show that a 16 bit arithmetics is suitable throughout the whole conversion system.

These characteristics suggest convenient hardware imolementations with low-cost arithmetic circuits.

\section{INIRODUCIION}

Efficient interfacing equipments between the frequency division multiplexing (FDM) format and the time division multiplexing (TDM) format (transmultiplexers) are of increasing interest for telephone networks, where the two multiplexing formats will have to coexist for a long time in the future.

In particular digital transmultiplexing methods are by far preferred because of the inherent advantages of the digital technology over analog techniques, their suitability for integration and the decreasing cost and increasing processing rate of digital integrated circuits (1-6).

Different structures have been proposed for the digital transmultiplexer implementation, as those based on the use of block processors (usually of the FrT type), per-channel approaches and multistage modulation tree-like structures (1-6).

This paper is concerned with the per-channel transmultiplexer structure and implementation, because they can attain a high modularity, an acceptable level of computational and control complexity and are convenient from the point of view of fault recognition and elimination (5). Furthermore these structures, when realized by means of finite- impulse-response (FIR) or wave digital filters, also guarantee an absolute system stability under the 10oped conditions, derived from the unbalanced four-wire/two-wire transitions (hybrids), that may occur in practice (5-7).

This paper considers a new per-channel approach to the transmultiplexer implementation, recently described in (8-9) and compared to other per-channel realizations in (10). This approach is based on the generation of a voiceband analytic signal (baseband SSB signal) and on its successive correct frequency allocation by appropriate digital interpolation and filtering for the conversion from the TDM to the FDM format. Operations in the reverse order also guarantee the inverse conversion (FDM to TDM) according to the network transposition nules (11). The method employs complex filters with relatively wide transition bandwidths and avoids the use of any product modulator. To satisfy the CCIIT specifications on the group delay of the conversion system, minimum-phase FIR complex filters are required. This paper, after briefly reviewing the method in Sect.2, describes the design of a bank of minimm-phase FIR complex filters for the proposed transmultiplexing method in sect.3. Based on these characteristics, the obtained performance of the designed transmultiplexer are reported in Sect. 4 and some implementation considerations are discussed in sect.5. As a specific application, this paper considers the transmultiplexer realization for the 60-108 kHz FDM 12-channel primary group, essentially for system simulation convenience and for comparison with other per-channel transmultiplexer solutions reported for the 12-channel case.

\section{TRANSMUULTIPIEXXR STRUCTURE}

The method generates a discrete analytic signal at the lower sampling rate $f=1 / T=8 \mathrm{kHz}$ (baseband SSB signal) which is successively allocated in the SSB format in one of the FDM channels at the sampling rate $\mathrm{Lf}_{\mathrm{S}}=\mathrm{L} / \mathrm{T}=112 \mathrm{kHz}$ for the $60-108 \mathrm{kHz}$ primary group. Essentially two complex filters are required to correctly allocate the digital SSB sig- 
nal in the ith channel of the FDis fonnat: a low rate $(8 \mathrm{kEz})$ complex filter $\bar{G}_{i}(\exp (j w T))$, w being the radian frequency, to generate the baseband SSB signal and a high rate $(112 \mathrm{kHz})$ complex channel filter $\vec{H}_{j}(\exp (j w T / L))$ for a correct positioning of the generated baseband $S S B$ signal in the desired FDM channel, through the process of aigital signal interpolation by the factor $\mathrm{L}=14$ and complex filtering. Ideally the two complex filters are defined for $i=1,2, \ldots, 12$ as (9) : .

$\bar{G}_{i}(\exp (j w T))=1+(-1)^{i}|\sin w T| / \sin w T$

and

$$
\bar{H}_{i}(\exp (j w T / L))=\left\{\begin{array}{l}
1, \text { if } s_{s} / 2 \leqslant f \leqslant(i+1) f_{s} / 2 \\
\text { undefined, }(i-1) f_{s} / 2 \leqslant f \leqslant \text { if } / 2 \\
\text { undefined, (i+1)f } f_{s} / 2 \leqslant f \leqslant(i+2) f_{s} / 2 \\
0, \text { elsewhere } \\
w=2 \pi f
\end{array}\right.
$$

periodic with period Lf .

However the practically Simited extension (300 to $3400 \mathrm{~Hz}$ ) of the frequency band of the telephone signals allows these filters to have more relaxed specifications. Indeed their in-band ripple, out$o E$-band attenuation and transition bands have only to comply with the specifications for transmultiplexing equipment compiled by the CCITI Recommendations G.791, G.792 and G.793.

The complex filters $G_{i}(\exp (j w i))$ and $\bar{H}_{j}(\exp (j w T / L))$ can be expressed through their conjugate symmetric parts $G_{i}(\exp (j w T))$ and $H_{i}(\exp (j w / L / L))$ respectivel $\frac{i}{y}$, and their conjugate aintisymmetric parts, $j G_{j}^{\prime}(\exp (j w T))$ and $j H_{i}^{\prime}(\exp (j w T / L))$ respectively, in the form: $\bar{G}_{i}(\exp (j w T))=G_{i}(\exp (j w T))+j G_{i}^{\prime}(\exp (j w T))$

$\vec{H}_{i}(\exp (j w T / L))=H_{i}(\exp (j w T / L))+j H_{i}^{\prime}(\exp (j w T / L))$

Since the conjugate symmetric parts and the conjugate antisymetric parts are the Fourier transform of the reals parts and the imaginary parts, respectively, of the complex impulse respon-ses of $\bar{G}_{i}(\exp (j w T))$ and $\bar{H}_{i}(\exp (j w T / L))$, the transfer functions $G_{j}(\exp (j w T))^{i}, G_{i}^{\prime}(\exp (j w i))$, $H_{i}(\exp (j w T / L)),{ }_{H}^{\prime}(\exp (j w T / L))^{i}$ all correspond to real digital filters.

It is possible to show (9) that the correct allocation of the ith TDM signal, with spectrum $S_{i}(\exp (j w L))$, in the ith FDM channel in the required SSB modulation format can be expressed as

$$
\begin{aligned}
& Y_{i}(\exp (j w T / L))=S_{i}(\exp (j(w T+i \pi))) \cdot \\
& {\left[G_{i}(\exp (j w T)) H_{i}(\exp (j w T / L))-G_{i}^{\prime}(\exp (j w T)) \cdot\right.} \\
& \left.H_{i}^{\prime}(\exp (j w T / L))\right]
\end{aligned}
$$

where the operation $S_{i}(\exp (j(w T+i m)))$ on the input signal spectrum simply implies an altemate sign inversion of the signal samples at each odd channel input. The final digital FDir signal $Y(\exp (j w / / L))$ is obtained by suming all the $Y_{i}(\exp (j w T / L))$.

The transmultiplexer block diagram according to this method is shown in Fig. 1.

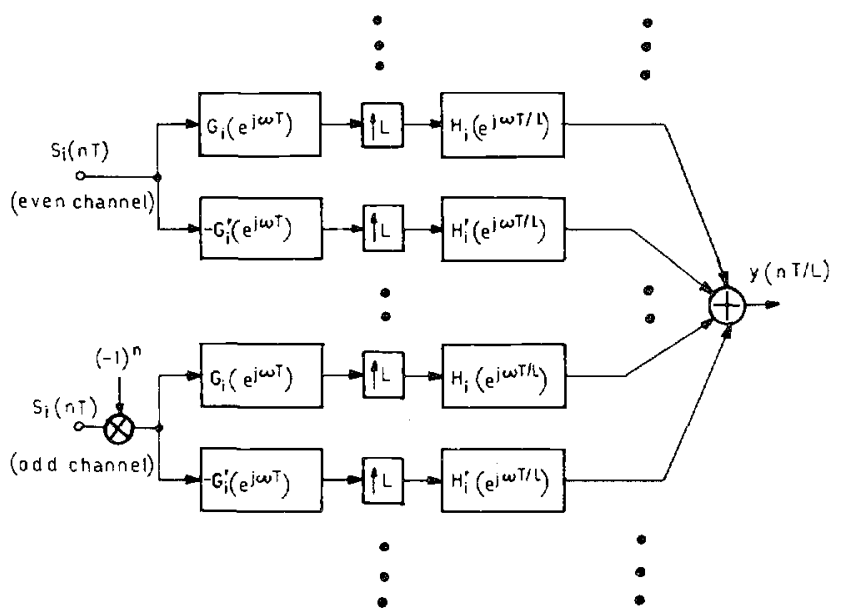

Fig.1. Block diagram of the conversion system

This conversion system allows relatively wide transition bands to the high rate filters, whose realization more heavily influences the overall system computational complexity. The two signal paths for each channel, according to (5), can be interpreted as performing the required SSB signal allocation by means of an in-band signal phase addition and phase cancellation of the signals in the two adjacent bands. Finally it should be observed that the number of different low rate filters $\vec{G}_{i}(\exp (j w T))$ is actually two, i.e. one for the odd channels and one for the even channels, although for notation convenience an explicit reference to the channel index $i$ has been retained.

\section{DESIGN OF IMINIMUM-PHASE FIR COMPLEX FILTERS}

To design FIR minimum-phase complex filters, a generalization of the method proposed in (12) (13) was developed. For a minimum-phase complex filter of length $\mathrm{N}$, a linear-phase equiripple complex prototype of length $M=2 \mathrm{~N}-1$ is firstly designed, using a modified version of the Parks-McClellan program (14). Its impulse response is complex conjugate with respect to the center coefficient and therefore the (real) frequency response of the noncausal filter, referred to the symmetry center, is $\mathrm{H}(\exp (j \omega))=\mathrm{a}(0)+\sum_{n=1}^{N-1}[a(n) \cos (n \omega)+b(n) \sin (n \omega)]$ $w$ being the angular frequency normalized to the filter sampling frequency. Obviously $a(n)$ and $b(n)$ are related to the actual non-causal filter coefficients $h(n)$ and, in particular, $a(0)=h(0)$.

Hence adding a quantity $x$ to $h(0)$, the frequency response (6) shifts upside by the same quantity. When $x$ varies from 0 to the maximum stopband deviation $\delta$, the zeroes on the unit circle of the 
Iinear-phase filter transfer function get closer. to each other and when $\mathrm{x}=\delta$ they become double zeroes on the unit circle.

Because if $z=z$ is a zero of the filter also $z_{0}^{*-1}$ (where * indicates complex conjugation) is a zero, the transfer function of the "shifted" filter can be expressed as

$\mathrm{H}_{\delta}(\mathrm{z})=\stackrel{*}{h}(\mathrm{~N}-1) \mathrm{z}^{-2(\mathrm{~N}-1)} \prod_{k=1}^{N-1}(\mathrm{z}-\mathrm{z} \mathrm{ok})\left(\mathrm{z}-\mathrm{z}_{\mathrm{ok}}^{*-1}\right)$

with $\left|z_{o k}\right| \leqslant 1$, for $k=1, \ldots, N-1$.

Following the same derivation as in (12), it is straightforward to show that, within a scale factor, the magnitude of the transfer function of the minimum-phase filter is given by $|\mathrm{H}(\exp (j \omega))|=$ $\left|H_{\delta}(\exp (j \omega))\right|^{\top / 2}$, with a corrisponding $z$-transfer function:

$H(z)=\sqrt{H^{*}(N-1) / \prod_{k=1}^{N-1} z_{o k}^{*}} z^{-(N-1)} \prod_{k=1}^{N-1}\left(z-z_{o k}\right)$

Finally, to approximate to 1 the filter passband response, the transfer function (8) must be scaled by the factor $(1+\delta)^{-172}$.

It is also easy to show that, in this case too, starting from a linear-phase equiripple prototype with in-band ripple $\delta_{1}$ and out-of-band ripple $\delta_{2}$, the corrisponding ripples $\delta_{1}^{\prime}$ and $\delta_{2}$ of the obtained minimum-phase filter are given by:

$\delta_{1}=\left(1+\delta_{1} /\left(1+\delta_{2}\right)\right)^{1 / 2}-1$

$\delta_{2}=\left(2 \delta_{2} /\left(1+\delta_{2}\right)\right)^{1 / 2}$

Hovever, the computer implementation of the outlined method, even in presence of exact equiripple ptototypes, presents many problens, due to the extreme difficulty of a numerical accurate determination of double zeroes. A computationally more convenient approach is the development of the method proposed in (13) for real filters to complex coefficients filters. A quantity $x$ greater (of the order of $20 \%$ ) than the stopband deviation $\delta_{2}$ of the linear-phase prototype is added to its center coef.ficient. Thus the stopband zeroes (double zeroes for $x=\delta_{2}$ ) split and move perpendicularly off the unit circle. Now the procedure finds all the prototype zeroes, retaining only those with magnitude less than 1. The minimum-phase filter is then synthetized by keeping the passband zeroes and setting to 1 the magnitude of the stopband zeroes. The transfer function of the synthetized minimum-phase filter is finally scaled by $(1+x)^{-1 / 2}$. It must be noted that, in this case, formulas (9) are only approximately valid.

\section{PERFORMANCE OF THE DESIGNED TRANSMULTIPLEXER}

The complex filters $\bar{G}_{j}(\exp (j w T))$ and $\overline{\mathrm{H}}_{i}(\exp (j w \mathrm{~T} / \mathrm{L}))$ were designed in order to satisfy the CCITT recomendations for transmultiplexer equipment.

In particular the group delay characteristics require a minimum-phase design for the low rate filters $\bar{G}_{i}(\exp (j w T))$ (9). They were designed accor- ding to the procedure described in the previous section and turned out to be 28-order FIR filters (29 coefficients), having the following characteristics (Fig.2 for even channels) : in-band deviation of $.19 \mathrm{~dB}$ and out-of-band minimum attenuation of $65.73 \mathrm{~dB}$, with a 15 bit coefficient accuracy.

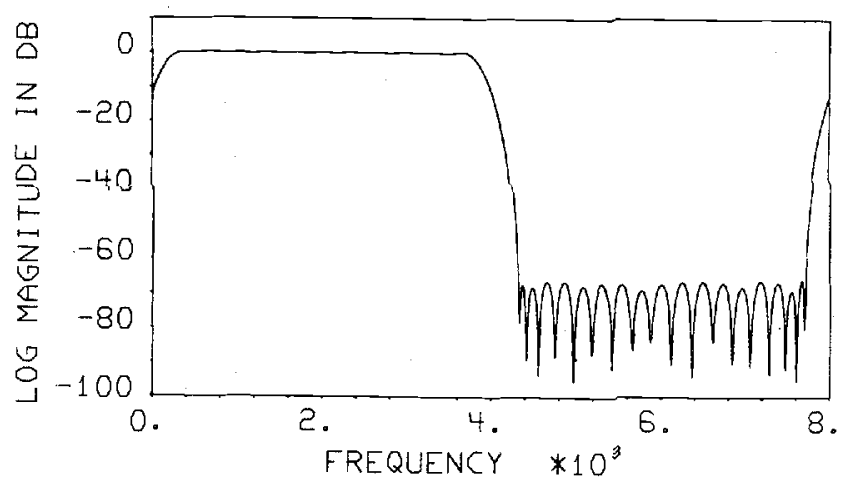

Fig.2. Frequency response of the minimumphase filter $\vec{G}_{i}(\exp (j w T))$ (even channels)

The filters $\bar{H}_{i}(\exp (j w T / L))$, designed as Iinear-phase filters due to their small contribution to the group delay (9), have the following characteristics (Fig.3): 68th order, in-band deviation .05 dB and out-of-band minimu attenuation greater than $65 \mathrm{~dB}$ for all the bank of filters, with a 12 bit coefficient accuracy.

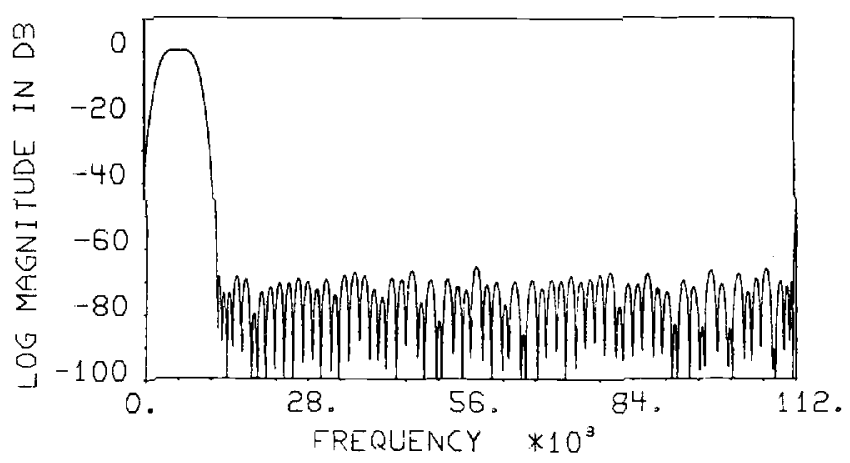

Fig.3. Frequency response of the 1st channel filter

The group delay distortion for each channel is shown in Fig.4, with superimposed the limits of the group delay characteristics derived for a single conversion from the CCITT recomendations.

A theoretical calculation of the noise performance shows (9) that a 16-bit arithmetic wordlength is sufficient for a fixed - point system implementation, to obtain an output noise variance of the same order than the variance of the quantization noise associated to the 8-bit A-law coded input signal. The computer simulations, carried out using a continuous input signal spectrum of a triangular form, confirmed the theoretical analysis. An example is shown in Fig.5, where only. the first channel 
is active.

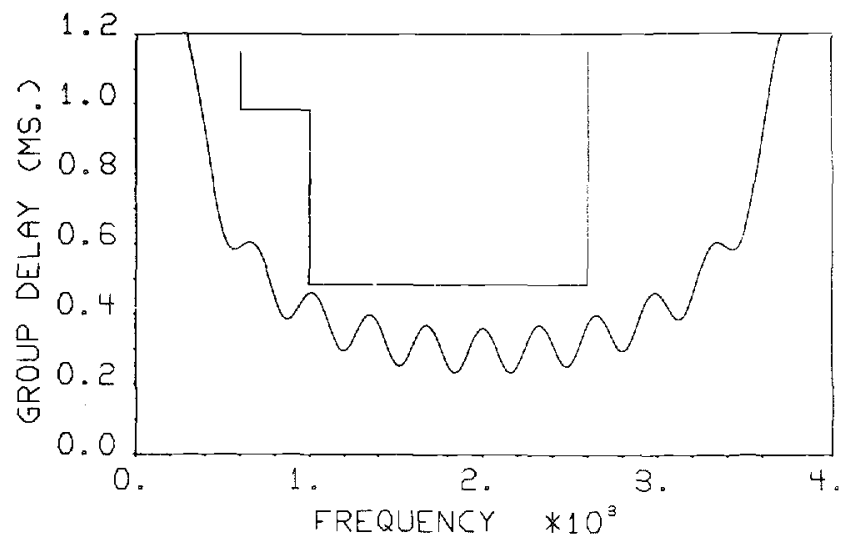

Pig.4. Group delay of the minimm-phase filter

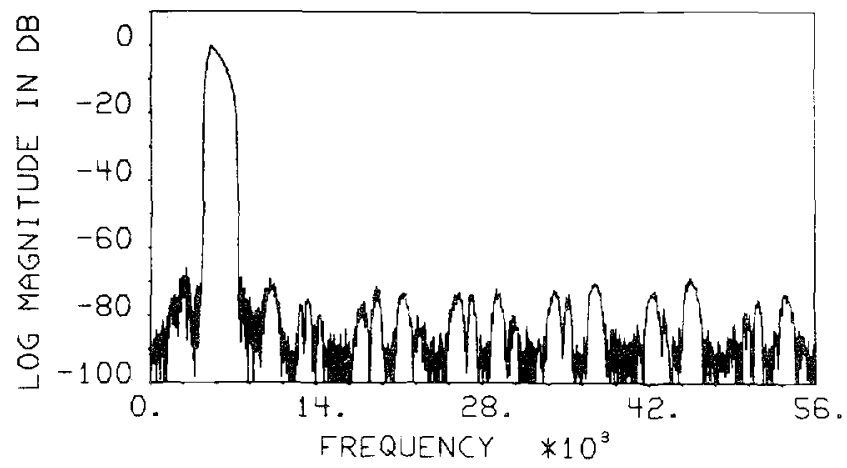

Fig.5. Simulation of the performance of the conversion system (first channel active)

\section{IMPLEMENTATION CONSTDERATIONS}

Because the channel filters $H_{j}(\exp (j w L / L))$ and $\mathrm{H}^{\prime}(\exp (j w T / L))$ interpolate their input signal by a factor $L=14$, they require $(69+1) / 14=5$ multiplications per output sample. Therefore the overall multiplication rate per channel amounts to

$\mathrm{R}_{\mathrm{M}}=2 \times(29 \times 8+5 \times 112) \times 10^{3}=1.584 \times 10^{6} \mathrm{mults} / \mathrm{s} / \mathrm{ch}$ (10)

This multiplication rate is comoarable to other per-channel approaches (1), with the inherent advantages of using FIR filters: stability, absence of limit cycles, reduced effect of the quantization errors and simpler implementation structures.

Moreover a high modularity is attained, because four filters with comparable computational complexity are required for each channel in the proposed structure. This means that the overall system can be implemented by using a unique building block, which realizes a FIR filter structure in direct form. The computational complexity of the faster filter allows the building block to be implemented by using present low cost simple serialparallel multipliers and, in perspective, also by single chip signal processors.

\section{CONCIUSIONS}

A per-channel solution to the transmaltiplexer problem has been developed, exploiting the properties of the analytic signal and using FIR digital filters.

In particular the design of minimm-phase complex filters is necessary to meet the CCITT specifications on the frequency response and the group delay and to achieve the required matching of the frequency response of the real filters in the two different channel paths. A generalization of known techniques to design FIR complex minimum-phase digital filters has been developed and the performances of the system have been presented, showing the feasibility of the proposed system modular structure by means of simple arithmetic circuits.

\section{References}

(1) S.L.Freeny, R.B.Kieburtz,K.V.Mina, S.K. Tewksbury, "Systen Analysis of a TDM-EDM Translator/Digital A-type Channel Bank", IFFE Trans.Corm.Techn. COM-19,12:Dec. 1971.

(2) 11.G.Bellanger, J.L.Daguet, "TDM-FDM Transmultiplexer: Digital poliphase and FF"T", IEFE Trans. Comm. Techr. , co:1-22, 9: Sept.1974.

(3) IEEE Trans. Communications, Special Issue on Digital Signal Frocessing, COM-26,5:May 1978.

(4) Conf.Rec. ICC (Denver, Co), 1981, Sessions 7 \& 18.

(5) H.Scheuermann, H.Gökler, "A Comprehensive Survey of Digital Transmiltiplexing Methods", Proc. IEEE, Vol.69,11: Nov.1981.

(6) IFFE Trans. Communications, Special Issue on Transmultiplexers, to be published.

(7) A.Fettweis, K. Meerkötter, "On Parasitic Oscillations in Digital Filters under Iooped Conditions", IEEE Trans.Circuits Syst, CAS-24,9:Sept1977.

(8) E.Del Re, "A New Approach to Transmultiplexer Implementation", Conf.Rec. ICC (Denver, Co), 1981.

(9) E.Del Re,P.L.Emiliani, "An Analytic Signal Approach for Transmultiplexers:Theory and Design", IEEE Trans. Communications, to be published.

(10)E.Del Re,F.Ronconi, P.Salvi, P.Semenzato, "Comparison of non-FF"T Methods of TDM-FDM Transmultiplexing",Alta Frequenza, Vol. LI, 1:Jan.1982.

(11) T.A.C.M.Claasen,W.F.G.Ikecklenbräuker, "on the Transposition of Linear Time-varying Discretetime Networks and its Applications to Multirate Digital Systems", Philips J.Res.,33,n.1/2:1978.

(12) O.iferrmann, H.W.Shuessler, "Design of Non-recursive Digital Filters with Minimum Phasei",Electron. Lett., 6, 1970.

(13) H.Ueda, T.Aoyama, "A Practical Procedure for Designing FIR Filters with optimm Magnitude and Ni-.nimum Phase", in Proc. 1979 ISCAS, Tokio, Japan.

(14)M.T.McCallig, "Design of Digital FIR Filters with Complex Conjugate Pulse Responses" , IEEF Trans. Circuits Syst.,CAS-25,12:Dec 1978. 